\title{
A generalized multilevel-hybrid chaotic oscillator for low-cost and power-efficient short-range chaotic communication systems
}

\author{
Ammar Dukhan ${ }^{1,2^{*}}$, Dhammika Jayalath ${ }^{1}$, Peter van Heijster ${ }^{1}$, Bouchra Senadji ${ }^{1}$ and Jasmine Banks ${ }^{1}$
}

\begin{abstract}
In this paper, we present and evaluate a novel multilevel hybrid-chaotic oscillator. The proposed generalized multilevel-hybrid chaotic oscillator (GM-HCO) was created by combining a multilevel discrete function generated from user data with a continuous function having a damping factor greater than $\ln (2)$ to achieve variable rates and adaptive carrier frequencies. Improved spectral efficiency and lower complexity of the transceiver compared with differentially coherent systems were achieved by multilevel signals at the transmitter and a matched filter at the receiver. An exact analytical solution for the generalized fixed basis function and the impulse response of the matched filter were also derived. The bit error rate (BER) expression of the GM-HCO was derived for two levels. It was found that the noise performance of the proposed system was better than a hybrid chaotic system based on forward time and differential chaos shift keying (DCSK). A comprehensive set of simulations were carried out to evaluate the performance of the proposed system with chaotic communication systems in the presence of additive white Gaussian noise (AWGN). The performance of the proposed system was comparable with that of conventional communication systems. The results demonstrate that the proposed system can offer better noise performance than existing chaotic communication systems, and it also offers variable transmitter frequencies and improved spectral efficiency. Noise-like behavior of the chaotic signals provides an additional layer of security at the physical layer compared with conventional (sinusoidal) communication systems.
\end{abstract}

Keywords: Chaotic signal, Discrete function, Hybrid chaotic system, M-ary modulation, Multilevel system, Matched filter, Generalized multilevel hybrid chaotic oscillator, Reverse time, Forward time

\section{Introduction}

Existing frequency up/down conversions and power amplification stages in conventional wireless communication systems increase the costs of the design, demand more space and weight, and consume more energy. Moreover, non-linear power amplification stages distort the base-band signal and degrade the quality of the signal. These are important challenges for wearable and internet of things (IoT) devices and sensor networks. It has been shown that these drawbacks of conventional systems

\footnotetext{
*Correspondence: ammar.moufak@icloud.com

This article is published in 27th International Telecommunication Networks and Applications Conference (ITNAC), November, 2017 in part.

'Science and Engineering Faculty, Queensland University of Technology, 2 George St., Brisbane, Australia

${ }^{2}$ Electrical Engineering Department, College of Engineering, Al-Mustansiriya University, Palestine St., Baghdad, Iraq
}

can be overcome by using chaotic signals that can be created by unsophisticated circuits without frequency conversions and power amplification stages [1, 2]. Chaotic signals with low-complexity transceivers and low -power consumption provide an attractive solution to the shortrange low-power communication challenge. Although chaotic signals possess many features of stochastic processes, they are always generated by simple deterministic dynamical systems. Significant features, such as aperiodic long-term behavior, deterministic nature, and sensitivity to initial conditions make chaotic signals a perfect candidate to realize wireless communication systems with simple transceiver implementations.

In the past, it was believed that chaotic behavior could be observed only in complex systems, such as neurology $[3,4]$, mechanics [5, 6], fluid dynamics [7], and oceanography [8]. In 1963, however, Eduard Lorenz [9] overturned 
existing dogma in this field, by simulating weather patterns using three simple equations. It was a breakthrough and the beginning of a new notion and development of deep insights into expressing highly complex chaotic behavior using simple equations. Since then, the representation of complex systems including living $[10,11]$ and non-living systems $[12,13]$ has become achievable.

Initial research involving chaotic communication has focused on mapping information into a chaotic signal and recovering it successfully at the receiver. In the 1990s, Pecora and Caroll [14] observed that chaotic signals could be synchronized successfully. Later, Cuomo and Oppenheim [15] designed the first practical communication scheme based on chaotic signals. After the seminal work by Pecora and Carroll, who provided the opportunity to use chaotic signals in communication systems, researchers around the world were quick to propose various chaotic communication systems. Chaos shift keying (CSK) [16], differential chaos shift keying (DCSK) [17], chaotic on-off-keying (COOK) [18], and frequency modulation-differential chaos shift keying (FM-DCSK) [18] in single input single output (SISO) and multiple input and multiple output (MIMO) channels are some of the popular chaotic communication systems that have been proposed to date.

Unfortunately, these aforementioned chaotic communication systems are impractical for real applications for two main reasons. First, there are no practical chaos synchronization methods that can achieve perfect synchronization for coherent receivers, such as CSK and COOK, as it is hard to produce two physically identical chaotic circuits for drive and response synchronization techniques [19]. Second, it is difficult in practice to implement delay line circuits with ultra-wide band (UWB) in some chaotic communication systems, such as DCSK and FM-DCSK [20]. Furthermore, these systems have low-power efficiency and low throughput in general. Several studies aiming at solving these issues to increase the throughput and the power efficiency can be found in the literature [21-23]. However, these solutions have sophisticated designs and synchronization issues in internal circuits. Their noise performance is also inferior to conventional systems, such as binary phase shift keying (BPSK). Numerical approximations used for chaotic maps or attractors also contribute to performance degradation compared with the conventional systems which are based on analytical solutions.

The pioneering work of Corron et al. led to the realization of chaotic oscillators (dynamic systems) with exactly solvable differential equations by combining discrete systems with continuous systems [24, 25]. These differential equations are 2nd order. They are called hybrid chaotic systems which can topologically represent an attractor, such as the Lorenz attractor [9] or the Rossler attractor
[26]. For instance, Corron, et al. [27] synthesized a chaotic waveform to mimic two different chaotic attractors that depend on the state of the discrete system. Hybrid chaotic systems can be written as a linear convolution of binary symbols and a fixed basis function and produce an exactly solvable system as elucidated by Corron in 2010 [28]. These signals can be coherently detected at the receiver using a filter matched to the basis function $[28,29]$. As a result, hybrid chaotic systems have attracted a great deal of attention in the wireless communication system field due to a simple transceiver implementation [30-33] and better noise performance [28]. For example, Corron and Blakely [34] used the hybrid chaotic system to derive an equation of a matched filter to realize a better noise performance than previous chaotic communication systems and a performance closer to conventional BPSK communications. Recently, Milosavljevic et al. [35] presented a hybrid chaotic system with multilevel discrete states introducing a first -order equation for the first time in [36]. However, their system is based on forward time, and they have not evaluated its performance under AWGN and Rayleigh channels.

Limitations of hybrid chaotic systems in [28, 34] force them to operate at low-throughput levels and operating frequencies that are lower than the acoustic frequency boundary [35, 37-39] because they are based on forward time and symbolic dynamics. Although hybrid chaotic systems based on reverse time have solved the problem of low-operating frequencies, presented a simpler circuit, and provided low-cost and low-power consumption compared with hybrid chaotic systems based on forward time [24, 40-42], they have not been evaluated with multilevel discrete states under AWGN. Therefore, our contributions can be summarized as:

- Designing a chaotic communications system using a multilevel $(M$-ary) approach to increase the throughput with the ability to operate at high carrier frequencies, low-cost, low-power consumption, and a simple circuit.

- Presenting a generalized multilevel-hybrid chaotic oscillator (GM-CHO) based on reverse time with a generalized fixed basis function which will be used at the receiver side to improve and detect chaotic signals, and evaluating its performance with an AWGN channel.

- Deriving the bit error rate expression of the GM-HCO based on a generalized fixed basis function for two levels, to emphasize that the theoretical and simulated results match under AWGN channel conditions.

The rest of the paper is organized as follows. In Section 2, we propose the generalized multilevel-hybrid 
chaotic oscillator and present its corresponding chaotic map and the matched filter receiver. The bit-error rate of GM-HCO is derived in Section 3. Section 4 contains the results, and the discussion is presented in Section 5 . Section 6 concludes the paper.

\section{Proposed generalized multilevel-hybrid chaotic oscillator}

In this section, we discuss the adoption of a hybrid chaotic system based on reverse time because it does not depend on the symbolic dynamic concept, and it can be used to achieve $M$-ary modulation at varying frequencies and at low -design cost compared with a hybrid chaotic system based on forward time. It is comprised of a continuous function, $u(t) \in \mathbb{R}$, and a discrete function, $s(t)$. Traditionally, multilevel phase and frequency modulation cannot be achieved with chaotic communications due to the inherent non-periodic nature of the chaotic signal. However, a multilevel amplitude modulated chaotic communication system can be implemented with the help of the proposed hybrid chaotic signal oscillator. For $M$-ary amplitude modulation, $M=2^{b}$ where $M$ is the number of amplitude states in the constellation and $b$ is the number of data bits required to specify a given amplitude state. Although the idea of this new approach is similar to antipodal BPSK in terms of polarity, the Euclidean distance between the two message points in this approach is different depending on the value of the discrete states. The differential equation of the proposed hybrid chaotic oscillator is given by:

$$
u^{\prime \prime}(t)+2 \beta u^{\prime}(t)+\left(\omega^{2}+\beta^{2}\right) u(t)=\left(\omega^{2}+\beta^{2}\right) s(t)
$$

where $\beta$ and $\omega$ are fixed parameters. This is a 2nd-order linear non-homogeneous differential equation taken from $[24,41]$, and it is considered to be a non-autonomous dynamic system. However, since the discrete function has a random behavior which depends on the data information, it will make the differential equation behave chaotically in reverse time. In particular, $s(t)$ will be:

$$
s(t)=m_{i}, \quad(i-1) T_{s} \leq t<i T_{s},
$$

for $i \in \mathbb{N}_{>0}$ and $m_{i} \in\left\{ \pm M_{j} \in \mathbb{R} \mid j=1,2, \ldots, N\right\}$, where $N$ is potentially infinite and $\pm M_{j}$ are potential amplitudes of the signal chosen in such a fashion to ensure that there is no interference in energy between different amplitudes. The period $T_{s}$ of the symbol is chosen so that the transition between different amplitudes will be achieved when the derivative of the continuous function reaches zero. That is, $u^{\prime}\left(i T_{s}\right)=0$. Consequently, $T_{s}=$ $2 \pi / \omega$. Therefore, Eq. 2 is inherently different from the 2nd-order linear differential equations based on forward time in $[28,34]$ where they assumed that $s(t)=\operatorname{sgn}(u(t))$ to generate a random binary sequence. These equations depend on the symbolic dynamic concept where a discrete signal depends on the sign of a continuous signal. The symbolic dynamic concept cannot provide us with the ability to change the operating frequency, and it can only be used for coding data information, such as a pseudo-noise (PN) sequence generator that is used in code division multiple access (CDMA). However, the proposed Eq. 2 presents a novel multilevel discrete function which depends on the data information and works at multiple levels. The damping factor of the proposed oscillator is given by $\beta=f_{d} \ln (2)$, where $f_{d}=\frac{1}{T_{s}}$ is the damping frequency. The angular frequency $\omega$ of the proposed hybrid chaotic oscillator is $\omega=2 \pi f_{d}$; thus, as a consequence, $\beta \geq \ln (2)$. The solution for Eq. 1 on the time interval $\left[(i-1) T_{s}, i T_{s}\right), u_{g}^{i}(t)$, can be obtained by finding the homogeneous solution, $u_{h}^{i}(t)$, to the associated homogeneous problem:

$$
u^{\prime \prime}(t)+2 \beta u^{\prime}(t)+\left(\omega^{2}+\beta^{2}\right) u(t)=0
$$

and a particular solution, $u_{p}^{i}(t)$, where $u_{g}^{i}(t)=u_{h}^{i}(t)+$ $u_{p}^{i}(t)$. The homogeneous solution $u_{h}^{i}$ on $\left[(i-1) T_{s}, i T_{s}\right)$ is given by:

$$
u_{h}^{i}(t)=C_{1}^{i} e^{-\beta t} \cos (\omega t)+C_{2}^{i} e^{-\beta t} \sin (\omega t)
$$

where $C_{1}^{i}$ and $C_{2}^{i}$ are arbitrary constants of integration. The next step is to find the particular solution. Since the discrete function $s(t)$ is, by construction, constant on [ $\left.(i-1) T_{s}, i T_{s}\right)$, a particular solution on $\left[(i-1) T_{s}, i T_{s}\right)$ is also constant. A particular solution $u_{p}^{i}(t)$ of Eq. 1 on $\left[(i-1) T_{s}, i T_{s}\right)$ will be:

$$
u_{p}^{i}(t)=m_{i} \text {. }
$$

By combining Eqs. 4 and 5, the general solution, $u_{g}^{i}(t)$, on $\left[(i-1) T_{s}, i T_{s}\right)$ will be:

$$
u_{g}^{i}(t)=C_{1}^{i} e^{-\beta t} \cos (\omega t)+C_{2}^{i} e^{-\beta t} \sin (\omega t)+m_{i} .
$$

To determine the general solution $u_{g}(t)$ of Eq. 1, we have to ensure that the solutions $u_{g}^{i}(t)$, as well as their derivatives $\left(u_{g}^{i}\right)^{\prime}(t)$, match at the boundaries of each time interval. That is, $u_{g}^{i}\left((i-1) T_{s}\right)=u_{g}^{i-1}\left((i-1) T_{s}\right)$ and $\left(u_{g}^{i}\right)^{\prime}\left((i-1) T_{s}\right)=\left(u_{g}^{i-1}\right)^{\prime}\left((i-1) T_{s}\right)$, for $i=2,3, \ldots, N$. This recursively defines $C_{1}^{i}$ and $C_{2}^{i}$ for $i=2,3, \ldots, N$. After some algebra, we find $C_{1}^{i}=C_{1}^{1}+\sum_{j=1}^{i-1}\left(m_{j}-\right.$ $m_{j+1)} e^{j \beta T_{s}}$ and $C_{2}^{i}=C_{2}^{1}+\frac{\beta}{\omega} \sum_{j=1}^{i-1}\left(m_{j}-m_{j+1}\right) e^{j \beta T_{s}}$ for $i=2,3, \ldots, N$. This determines the general solution $u_{g}(t)$ of the hybrid chaotic oscillator given in Eq. 1. It can be used with Eq. 2 to generate $M$-ary modulation.

At $t=0, C_{1}$ and $C_{2}$ can be found by using two initial conditions $u_{g}^{\prime}(0)=0$ and $u_{g}(0)=u_{0}$. Then, the solution to Eq. 1 can be derived as:

$$
\begin{aligned}
\left(u_{g}\right)^{\prime}(t)= & -C_{1} \beta e^{-\beta t} \cos (\omega t)-C_{1} \omega e^{-\beta t} \sin (\omega t) \\
& -C_{2} \beta e^{-\beta t} \sin (\omega t)+C_{2} \omega e^{-\beta t} \cos (\omega t)+0,
\end{aligned}
$$


at $t=0$, this simplifies to:

$$
0=-\beta C_{1}+\omega C_{2} \Rightarrow C_{2}=\frac{\beta C_{1}}{\omega} .
$$

By substituting:

$$
\therefore u_{g}(t)=C_{1} e^{-\beta t}\left[\cos (\omega t)+\frac{\beta}{\omega} \sin (\omega t)\right]+m_{1},
$$

on the first time interval, at $t=0$, Eq. 7 will be:

$$
\begin{aligned}
& u_{g}(0)=u_{0}=C_{1} e^{0}\left[\cos (0)+\frac{\beta}{\omega} \sin (0)\right]+m_{1} \\
& u_{0}=C_{1}+s(0) \Rightarrow C_{1}=-s(0)+u_{0} .
\end{aligned}
$$

Since $s(0)=m_{1}$, we have $C_{1}=-m_{1}+u_{0}$, and $u_{g}(t)$ will be:

$$
\begin{array}{r}
u_{g}(t)=\left(u_{0}-m_{1}\right) e^{-\beta t}\left[\cos (\omega t)+\frac{\beta}{\omega} \sin (\omega t)\right]+m_{1} \\
\text { for } 0 \leq t<T_{s} .
\end{array}
$$

This is the solution for period $0 \leq t<T_{s}$. The solution for period $T_{s} \leq t<2 T_{s}$ is:

$$
\begin{array}{r}
u_{g}(t)=\left[e^{\beta T_{s}}\left(m_{1}-m_{2}\right)+\left(u_{0}-m_{1}\right)\right] e^{-\beta t} \times \\
{\left[\cos (\omega t)+\frac{\beta}{\omega} \sin (\omega t)\right]+m_{2} .}
\end{array}
$$

For period $2 T_{s} \leq t<3 T_{s}$, the solution is:

$$
\begin{array}{r}
u_{g}(t)=\left[e^{2 \beta T_{s}}\left(m_{2}-m_{3}\right)+e^{\beta T_{s}}\left(m_{1}-m_{2}\right)+\left(u_{0}-m_{1}\right)\right] \times \\
e^{-\beta t}\left[\cos (\omega t)+\frac{\beta}{\omega} \sin (\omega t)\right]+m_{3},
\end{array}
$$

and for period $3 T_{s} \leq t<4 T_{s}$, the solution is:

$$
\begin{array}{r}
u_{g}(t)=\left[e^{3 \beta T_{s}}\left(m_{3}-m_{4}\right)+e^{2 \beta T_{s}}\left(m_{2}-m_{3}\right)\right. \\
\left.+e^{\beta T_{s}}\left(m_{1}-m_{2}\right)+\left(u_{0}-m_{1}\right)\right] \times \\
e^{-\beta t}\left[\cos (\omega t)+\frac{\beta}{\omega} \sin (\omega t)\right]+m_{4}
\end{array}
$$

In general, for $(i-1) T_{s} \leq t<i T_{s}$, the solution can be expressed as:

$$
\begin{array}{r}
u_{g}(t)=\left[\sum_{j=2}^{i} e^{(j-1) \beta T_{s}}\left(m_{j-1}-m_{j}\right)+\left(u_{0}-m_{1}\right)\right] \times \\
e^{-\beta t}\left[\cos (\omega t)+\frac{\beta}{\omega} \sin (\omega t)\right]+m_{i} .
\end{array}
$$

This provides the exact solution of the proposed generalized multilevel-hybrid chaotic oscillator under the initial conditions $u(0)=u_{0}$ and $u^{\prime}(0)=0$, for all possible discrete states and $\beta \geq \ln (2)$. The basis function can be extracted from this equation, and it can be considered as the generalized fixed basis function of the matched filter. The generalized fixed basis function, $u_{b}\left(t ; u_{0}\right)$, is:

$u_{b}\left(t ; u_{0}\right)= \begin{cases}0, & \text { for } t<0, \\ \left(\frac{u_{0}}{m_{1}}-1\right) e^{-\beta t}\left(\cos (\omega t)+\frac{\beta}{\omega} \sin (\omega t)\right)+1, & \text { for } 0 \leq t<T_{s}, \\ \left(1+\left(\frac{u_{0}}{m_{1}}-1\right) e^{-\beta T_{s}}\right) \times & \\ e^{\beta\left(T_{s}-t\right)}\left(\cos (\omega t)+\frac{\beta}{w} \sin (\omega t)\right), & \text { for } t>T_{s} .\end{cases}$

Particularly, for $u_{0}=0$ and $m_{1}=1$, Fig. 1 shows a simulation of Eq. 9 and shows the generalized fixed basis function of GM-HCO for four periods of $T_{s}$.

Thus, the chaotic signal generated at the transmitter can now be obtained by convolving the generalized fixed basis function with the discrete states:

$$
u(t)=m_{1} u_{b}\left(t ; u_{0}\right)+\sum_{j=2}^{\infty} m_{j} u_{b}\left(t-T_{s}(j-1) ; 0\right)
$$

where $m_{j}$ is the amplitude of the discrete state that weights the generalized fixed basis function at the $j$ th time interval. Figure 2 shows the matching between the generalized fixed basis function in Eq. 10 and the differential equation in Eq. 1 with discrete signals $s(t)=$ $[-3,+1,-1,+3,+3,-3,-3,-3,+1,+1], T_{s}=1$

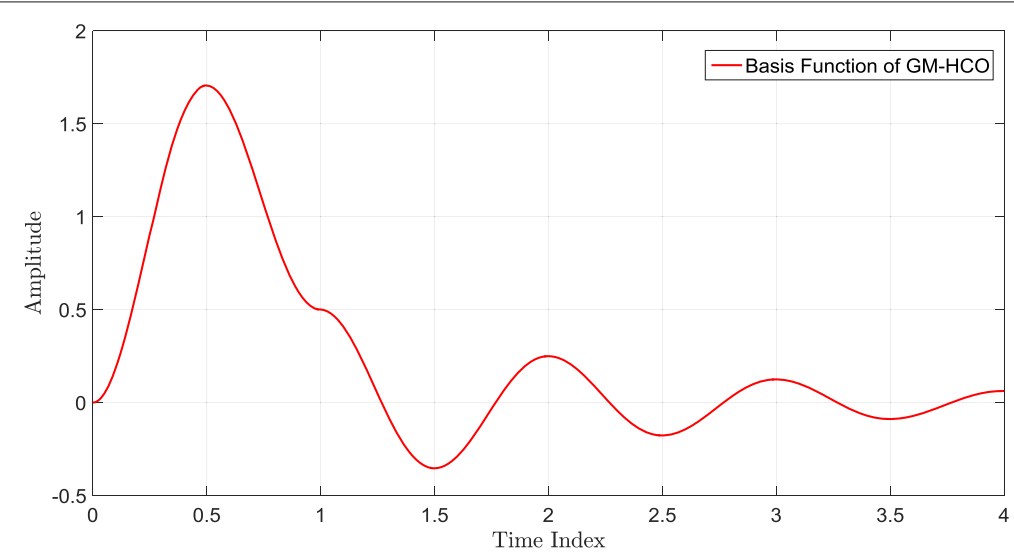

Fig. 1 Basis function, $u_{b}(t ; 0)$, of GM-HCO 


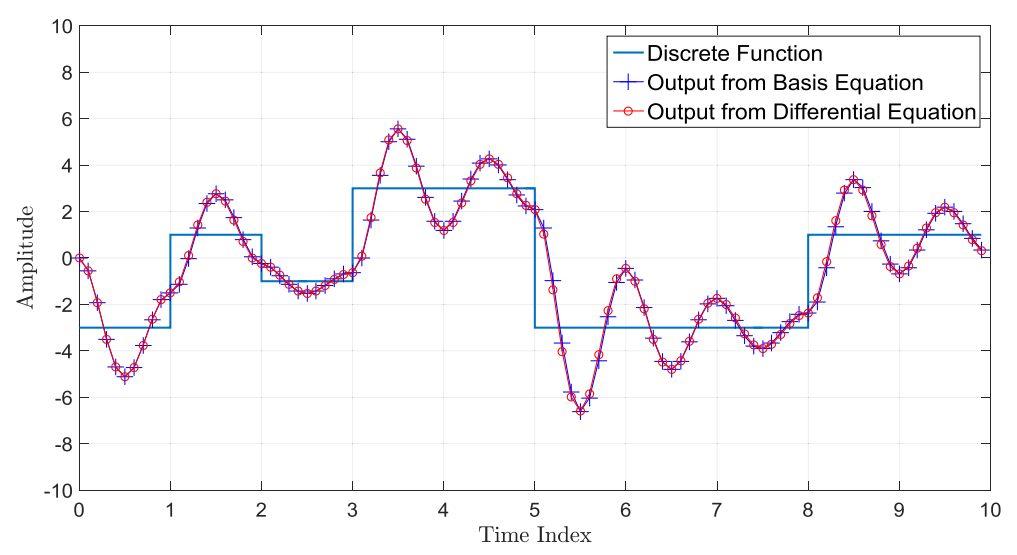

Fig. 2 Simulation of basis function and differential equation of GM-HCO

second and $\beta=\ln 2$. Equation 10 emphasizes that the behavior of chaotic signals in the reverse time can be expressed in terms of an exact analytic solution.

\subsection{Chaotic map of GM-HCO}

In this section, we derive a chaotic map of GM-HCO to emulate this behavior. Generally, a chaotic map can be represented by:

$$
X(t+1)=F\{X(t)\}
$$

where $F$ is a function that creates the mapping, and $X(t)$ is any time domain function. This map, which has a one-dimensional shape, can describe the system at some fixed time interval without a need to describe the system completely by using a differential equation. Therefore, at this point, the differential equation can be examined analytically. Therefore, let us recall Eq. 1 again and assume that the initial conditions are $u(0)=u_{0}, u^{\prime}(0)=0$, and $s(0)=s_{0}=m_{1}$.

A solution of this hybrid chaotic oscillator can be found in terms of $u_{0}$ and $s_{0}$. For the first time interval $\left[0, T_{s}\right]$, with $u_{g}(0)=u_{0}$ and $\left(u_{g}\right)^{\prime}(0)=0$, we have:

$$
u_{g}(t)=\left(u_{0}-m_{1}\right) e^{-\beta t}\left[\cos (\omega t)+\frac{\beta}{\omega} \sin (\omega t)\right]+m_{1},
$$

as derived in the previous section, and since $\omega=2 \pi / T_{S}$, at $t=T_{S}$ this simplifies to:

$$
u_{g}\left(T_{s}\right)=\left(u_{0}-m_{1}\right) e^{-\beta T_{s}}+m_{1}=: u_{1} .
$$

For the second time interval $\left[T_{s}, 2 T_{s}\right]$, we have:

$$
\begin{array}{r}
u_{g}(t)=\left[e^{\beta T_{s}}\left(m_{1}-m_{2}\right)+\left(u_{0}-m_{1}\right)\right] e^{-\beta t} \times \\
{\left[\cos (\omega t)+\frac{\beta}{\omega} \sin (\omega t)\right]+m_{2} .}
\end{array}
$$

By simplifying and substituting, we obtain:

$$
u_{g}(t)=\left(u_{1}-m_{2}\right) e^{\beta\left(T_{s}-t\right)}\left[\cos (\omega t)+\frac{\beta}{\omega} \sin (\omega t)\right]+m_{2},
$$

and, at $t=2 T_{s}$ :

$$
u_{g}\left(2 T_{s}\right)=\left(u_{1}-m_{2}\right) e^{-\beta T_{s}}+m_{2}=: u_{2} .
$$

In general,

$$
u_{g}\left(i T_{s}\right)=\left(u_{i-1}-m_{i}\right) e^{-\beta T_{s}}+m_{i}=: u_{i},
$$

for $i=1,2, \ldots$, and the general recurrence relation of the proposed map is:

$$
u_{n+1}=\left(u_{n}-m_{n+1}\right) e^{-\beta T_{s}}+m_{n+1} .
$$

Since Eq. 11 possesses a time reversible property, it can retain a positive Lyapunov exponent. Corron et al. in [24] stated that a reverse time chaos map can use the current state of the chaotic map to represent all of its past states. Therefore, Eq. 11 can be restated to find the positive Lyapunov exponent as follows:

$$
u_{n}=e^{\beta T_{s}}\left(u_{n+1}-m_{n+1}\right)+m_{n+1} .
$$

We can easily find that Eq. 12 is an iterated map. Therefore, by defining a mapping $F$ as:

$$
F\left(u_{n}\right)=e^{\beta T_{s}}\left(u_{n+1}-m_{n+1}\right)+m_{n+1},
$$

with derivative:

$$
F^{\prime}\left(u_{n}\right)=e^{\beta T_{s}} \text {. }
$$

Equation 13 is the slope of the map, and it is a constant for all $u_{n}$. The Lyapunov exponent, $\lambda$, of a onedimensional discrete map is defined as [43]:

$$
\begin{aligned}
& \lambda=\lim _{n \rightarrow \infty} \frac{1}{n} \sum_{i=0}^{n-1} \ln \left|F^{\prime}\left(u_{i}\right)\right|=\lim _{n \rightarrow \infty} \frac{1}{n} \sum_{i=0}^{n-1} \ln \left(e^{\beta T_{s}}\right)=\beta T_{s} . \\
& \text { Since } \beta=f_{d} \ln (2)=\frac{\ln (2)}{T_{s}}, \\
& \therefore \lambda=\ln (2) .
\end{aligned}
$$

Here, $\lambda$ is positive since $\beta$ is greater than zero. Therefore, since the iterated map is chaotic, the continuous time system is chaotic in backward time. 


\subsection{Matched filter receiver}

Since we have the generalized fixed basis function, the matched filter can easily be found. Traditionally, the impulse response of the matched filter, $h(t)$, will be the inverse time of the generalized fixed basis function (Eq. 9) for period $T_{s}$. Figure 3 shows the impulse response of the filter, $h(t)$, matched to the generalized fixed basis function with a damping factor $\beta=\ln 2$ and damping frequency of $1 \mathrm{~Hz}$ within $T_{s}=1$. The received signal will be:

$$
v(t)=u(t)+\eta(t)
$$

where $v(t)$ is the input of the filter, and it is corrupted by noise, $\eta(t)$. The output of the matched filter will be:

$$
\xi(t)=\int_{-\infty}^{T_{s}} h(t-\tau) v(\tau) d \tau .
$$

Since $v(t)$ is $u(t)+\eta(t)$,

$$
\xi(t)=\int_{-\infty}^{T_{s}} h(t-\tau) u(\tau) d \tau+\int_{-\infty}^{T_{s}} h(t-\tau) \eta(\tau) d \tau .
$$

This equation is a general convolution that can be used to recover the transmitted data at the receiver.

Some existing implementations of hybrid-chaotic systems utilizing symbol dynamics can be found in the literature [44]. In contrast, hybrid chaotic systems based on reverse time provide another insight when applying hybrid chaotic systems for high frequencies and a multilevel approach. Applications of the damping frequency in communication systems have come from the physical phenomena of the pendulum motion [45]. The data rate will determine the damping frequency, the damping factor, and the angular frequency. Figure 4 shows a proposed prototype of a novel communication system based on GM-HCO. As can be seen in this figure, the proposed system does not contain any up/down converters. Also, the noise-like behavior of the chaotic signals provides an additional layer of security at the physical layer. Therefore, security requirements at the upper layer could be relaxed, which further reduces the complexity and power consumption of the transceiver. Therefore, the proposed hybrid chaotic wireless communication system is a potential candidate for wearable devices, sensor networks, or IoT systems.

\section{Bit error rate performance of GM-HCO}

Let us suppose that we have two signals, $s_{1}(t)$ and $s_{2}(t)$, to be transmitted, and it is assumed that the received signals $r(t)$ are buried in AWGN, $n(t)$. The AWGN is a wide-sense stationary random process which has zero mean $\mu=0$ and variance $\sigma^{2}$, and it has a double-sided power spectral density $\frac{N_{o}}{2} \mathrm{~W} / \mathrm{Hz}$. Assuming equiprobable symbols, the bit error rate expression is given by:

$$
B E R=\frac{1}{2} Q\left(\frac{s_{o 1}-V_{T}}{\sigma_{o}}\right)+\frac{1}{2} Q\left(\frac{V_{T}-s_{o 2}}{\sigma_{o}}\right) .
$$

where $V_{T}$ is a comparator threshold voltage and $s_{O 1}$ or $s_{o 2}$ are output signals at sampling time, $T_{o}$. Since we have a fixed basis function of GM-HCO, we can design a linear time-invariant filter with an optimal comparator threshold voltage $V_{\text {opt }}$, so minimizing the probability of bit error will provide the following equation for the optimal comparator threshold voltage $V_{\text {opt }}$ :

$$
V_{o p t}=\frac{s_{o 1}+s_{o 2}}{2}
$$

where $V_{o p t}$ is greater than $s_{o 2}$ and less than $s_{o 1}$. Therefore, substituting the optimal value of threshold voltage of Eq. 17 into Eq. 16 yields:

$$
B E R=Q\left(\frac{s_{o 1}-s_{o 2}}{2 \sigma_{o}}\right)
$$

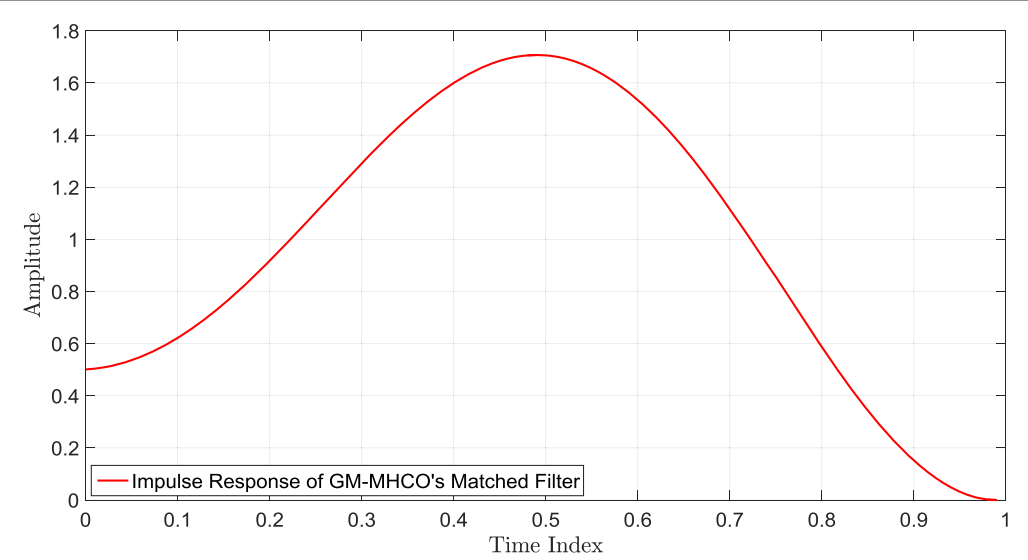

Fig. 3 Impulse response of the matched filter matched to the basis function given in Eq. 9 


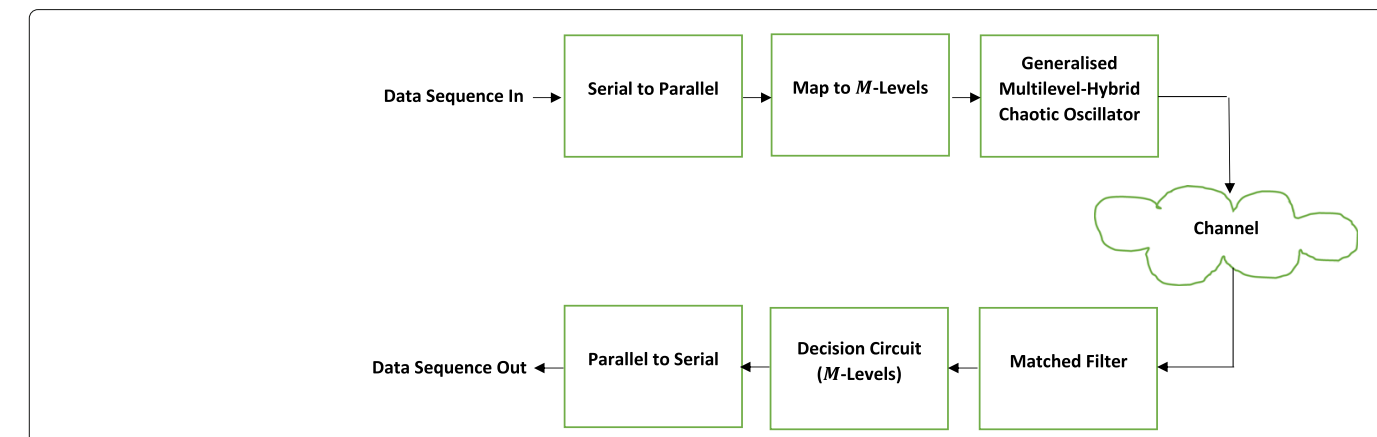

Fig. 4 Block diagram of the proposed hybrid chaotic wireless communication system based on GM-HCO

In the case of a matched filter, after optimizing Eq. 18, $d^{2}$ will become: the $B E R$ of a matched filter is given by:

$$
B E R_{M F}=Q\left(\sqrt{\frac{d^{2}}{2 N_{o}}}\right)
$$

where $d$ is the distance between two matched filter outputs, and it can be written as:

$$
\left.d^{2} \doteq s_{o 1}\right|_{t=T_{s}}-\left.s_{o 2}\right|_{t=T_{s}}
$$

where $h_{\text {opt }}(t)$ is a impulse response of the matched filter at sampling time $T_{S}$, which can be written as:

$$
h_{\text {opt }}(t)=s_{1}^{*}\left(T_{s}-t\right)-s_{2}^{*}\left(T_{s}-t\right)
$$

and $d^{2}$ will become:

$$
d^{2}=\int_{0}^{T_{s}}\left|s_{1}(t)-s_{2}(t)\right|^{2} d t
$$

From Eq. 8, and for the sake of simplicity, assuming the two probable binary \pm 1 for $0 \leq t<T_{s}$ :

$$
\begin{gathered}
u_{g_{+1}}(t)=-e^{-\beta t}\left[\cos (\omega t)+\frac{\beta}{\omega} \sin (\omega t)\right]+1 \\
u_{g_{-1}}(t)=e^{-\beta t}\left[\cos (\omega t)+\frac{\beta}{\omega} \sin (\omega t)\right]-1 .
\end{gathered}
$$

$$
d^{2}=\int_{0}^{T_{s}}\left|u_{g_{+1}}(t)-u_{g_{-1}}(t)\right|^{2} .
$$

Since the energy of each bit within $T_{s}$ is constant, $u_{g_{+1}}(t)=-u_{g_{-1}}(t)=u_{g}(t)$, and then $d^{2}$ can be written as:

$$
d^{2}=4 \int_{0}^{T_{s}}\left|u_{g}(t)^{2}\right|
$$

By substituting Eq. 20 into Eq. 19, the $B E R_{M F}$ of GM$\mathrm{HCO}$ is found to be:

$$
B E R_{G M-H C O}=Q\left(\sqrt{\frac{2 \int_{0}^{T_{s}}\left|u_{g}(t)^{2}\right|}{N_{o}}}\right)
$$

As can be seen in Eq. 21, the $B E R_{M F}$ of GM-HCO depends on the bit energy within one duration, $T_{s}$. In the special case, where we generate a binary sequence of \pm 1 , which alternates between -1 and +1 , we will obtain the exact result for BER of antipodal systems, such as BPSK, as shown in Fig. 5. In another special case, where we have two signals of 0 and 1, the BER of GM-HCO will be:

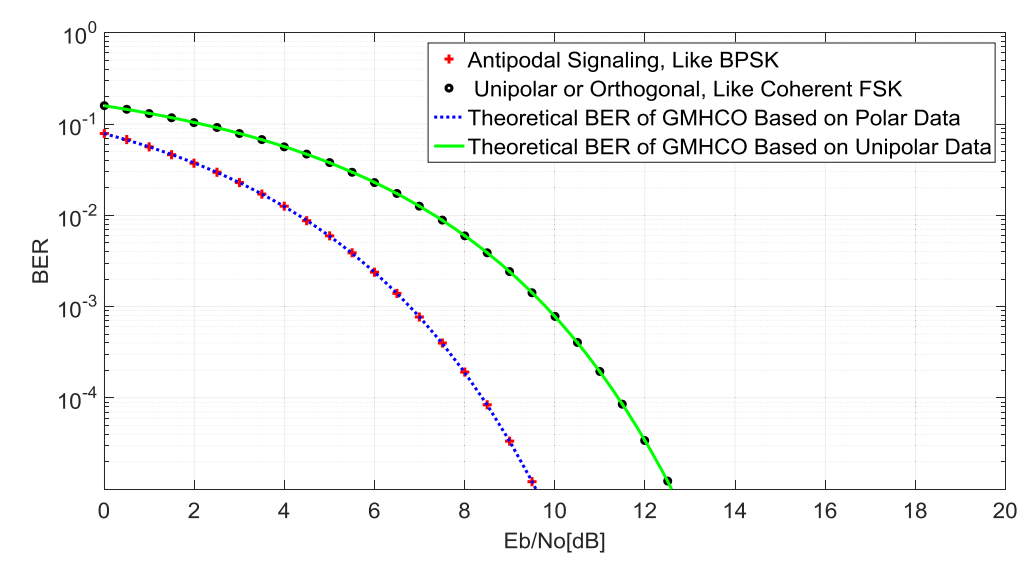

Fig. 5 The noise performance of GM-HCO 


$$
B E R_{G M-H C O}=Q\left(\sqrt{\frac{\int_{0}^{T_{s}}\left|u_{g}(t)^{2}\right|}{N_{o}}}\right)
$$

Equation 22 of GM-HCO is similar to unipolar or orthogonal signaling, as shown in Fig. 5. However, because the sequence of data is random, this will lead to degradation in noise performance by about $1 \mathrm{~dB}$ as shown in Fig. 6 . This figure also provides upper and lower boundaries for GM-HCO. This means if GM-HCO is driven by alternative sequence data of two symbols, this provides the best case noise performance which is matched to BPSK, while in the worst case, if it is driven by successive sequence data of one symbol. In the normal case, the BER of GM-HCO will be more complicated to estimate because of the problem of determining the bit energy. To clarify the problem of bit energy, the signal space representation can provide a deep insight into GM-HCO's behavior. Therefore, Fig. 7 presents the signal space of GM-HCO, thus as can be seen in this figure, we have one basis function, $\phi$, to represent the chaotic signal of GM-HCO. The shape of the signal space exhibits spiral behavior. This is due to the fact that the equation of GM-HCO contains an exponential term (damping factor) that causes the energy of the signal to decrease gradually if GM-HCO is driven by successive bits of a similar kind. The phase of GM-HCO's expression, $\theta$, can be 180 or zero because it depends on the sign of the level of the signal while the value of energy will vary and lead to degradation in noise performance. However, the best noise performance occurs when we have two levels $m_{i}$ of \pm 1 alternating, similar to antipodal signaling of conventional communication systems.

\section{Results}

In this section, we present an evaluation of the performance of the proposed hybrid chaotic wireless communication system in AWGN. For the sake of simplicity, the number of levels, $M$, is set to 4 , and discrete signals of \pm 3 and \pm 1 are chosen to study the dynamics of the new chaotic attractor using the one-dimensional chaotic map. Four important regions are chosen to illustrate the performance. A bit sequence is first mapped to symbols by a mapper based on the value of $M$ as illustrated in Fig. 4. Figure 8 shows the discrete state defined in Eq. 2 and the continuous behavior defined from the discrete state as described in Eq. 1. The black asterisks in the figure represent new values of the chaotic map defined for $\beta=10^{3} \ln (2)$ as defined by Eq. 11. Plotting these recurring asterisk points in Fig. 8 will draw the chaotic map of the proposed hybrid chaotic system as shown in Fig. 9. This figure shows the behavior of four levels in onedimensional space with $\beta=10^{3} \ln (2)$. The data rate for the damping frequency of $10^{3} \mathrm{~Hz}$ and four discrete levels is $\left(2 \times 10^{3}\right)$ bps. As a result, spectral efficiency is improved to $2 \mathrm{bits} / \mathrm{s} / \mathrm{Hz}$. Different values of damping factor also provide supporting evidence that the damping factor does not affect the shape or the behavior of the chaotic map, as shown in Fig. 10, which presents the chaotic map for $\beta=\ln (2), \beta=10^{3} \ln (2)$, and $\beta=10^{6} \ln (2)$.

Although a hybrid chaotic system based on reverse time has previously been able to achieve high frequencies [40, 44], a multilevel approach has not previously been applied. Thus, this new approach can provide an opportunity to employ chaotic signals in modern wireless communication systems with different frequencies and high transmission rates. The modification of discrete state in a hybrid chaotic system operates as an oscillator to provide a generalized fixed basis function and to implement a multilevel modulation technique. By recalling Eq. 1 and its derivative Eq. 2, 3D and 2D phase space projections for these levels can be drawn as in Figs. 11 and 12 .

In these figures, the four levels, \pm 1 and \pm 3 are very clear. Since the data rate is $2 \mathrm{kbps}$, the damping frequency will be $1 \mathrm{kHz}$, and the damping factor will be $\beta=10^{3} \ln (2)$.

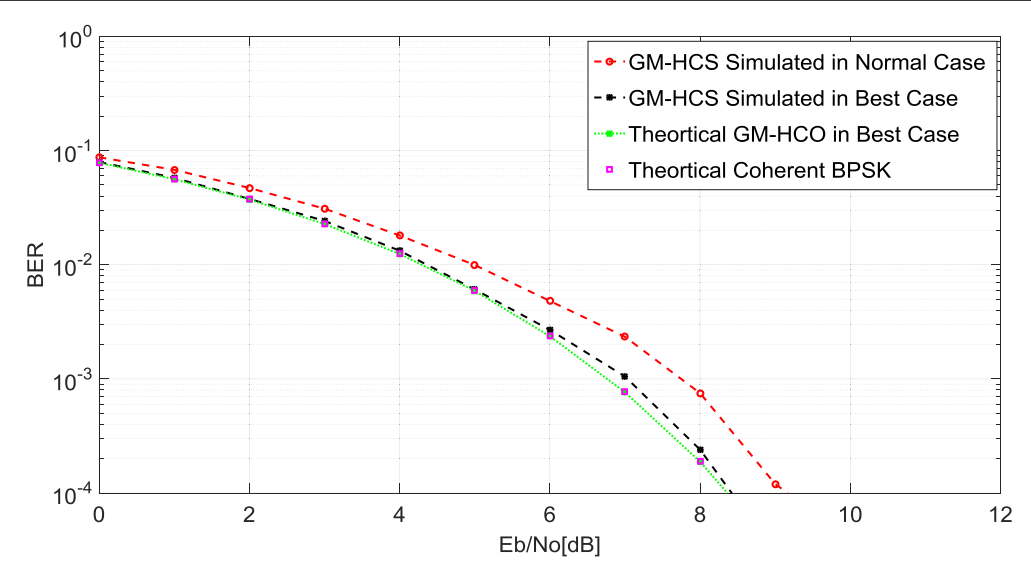

Fig. 6 The noise performance boundary of GM-HCO with data sequence of \pm 1 


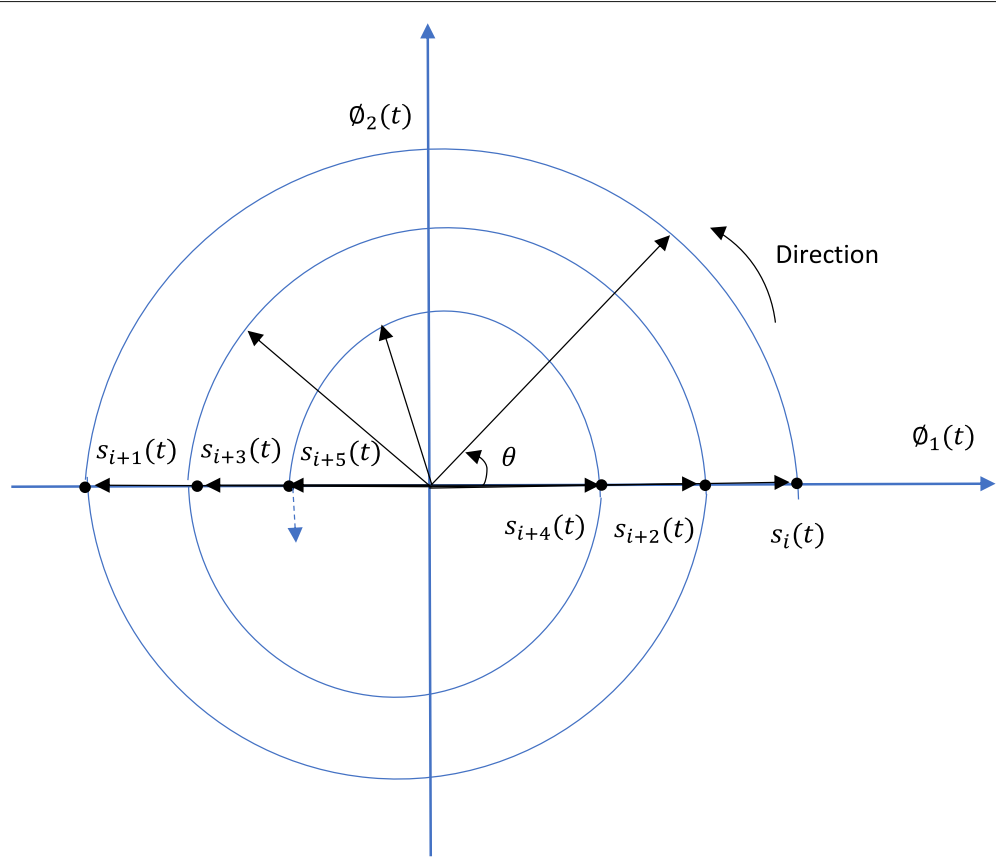

Fig. 7 Signal space representation of GM-HCO

A matched filter derived from the generalized fixed basis function of GM-HCO can extract the noisy signals received as in conventional communication systems. The threshold equation for each level will also be found by extracting the optimal values of the discrete states.

Thus, it is fairly straightforward that the symbols ( \pm 3 and \pm 1 ) can be extracted so that the two bits (symbols) can be recovered at the receiver side. The significant findings were carried out using the MATLAB software, and in these experiments, the timing problem was assumed to be solved. The shape of the transmitted signal can be seen in Fig. 8. Here, the chaotic signal carries two bits per 1 millisecond which represent one of four symbols $( \pm 3$ and \pm 1 ). Figure 13 presents the performance of the GM-HCO based on the generalized fixed basis function (Eq. 9) for two levels under AWGN channel conditions. As can be seen in this figure, there is a significant improvement in noise performance using

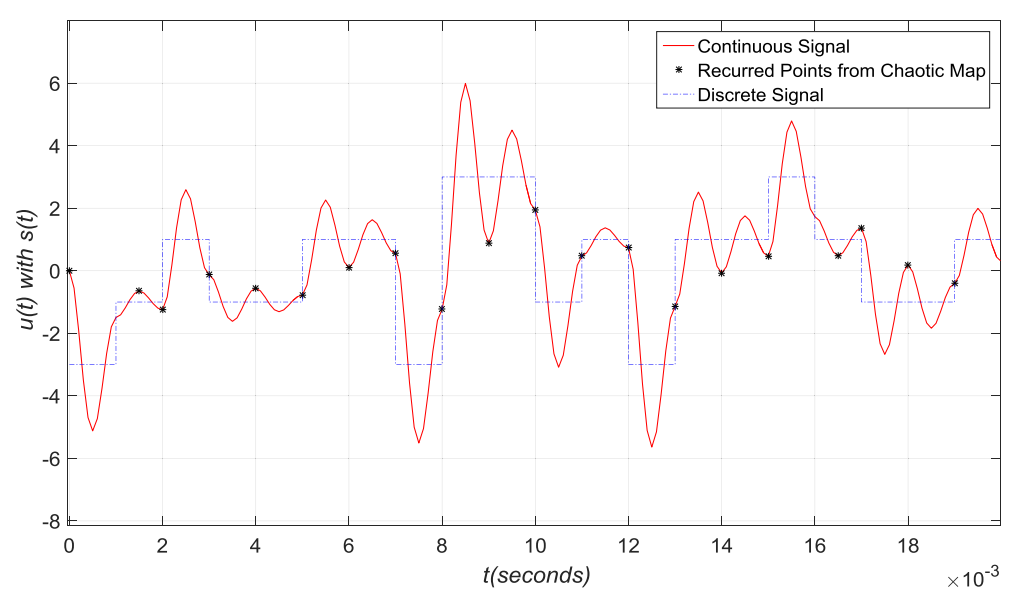

Fig. 8 Numerical simulation of proposed hybrid chaotic signal with $\beta=10^{3} \ln (2)$ 
Dukhan et al. EURASIP Journal on Wireless Communications and Networking

(2020) 2020:23

Page 10 of 14

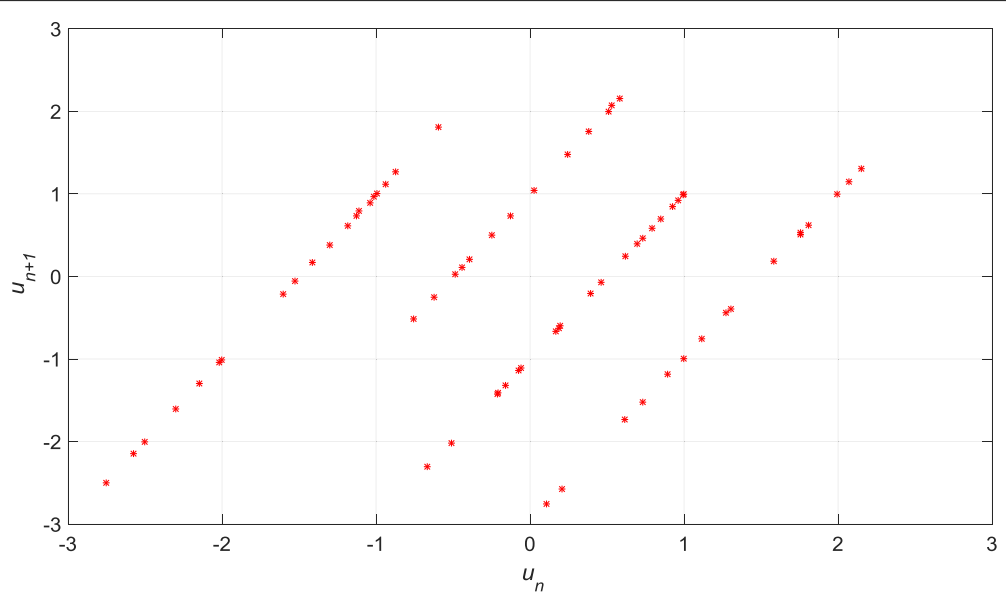

Fig. 9 A chaotic map of the proposed system with $\beta=10^{3} \ln (2)$

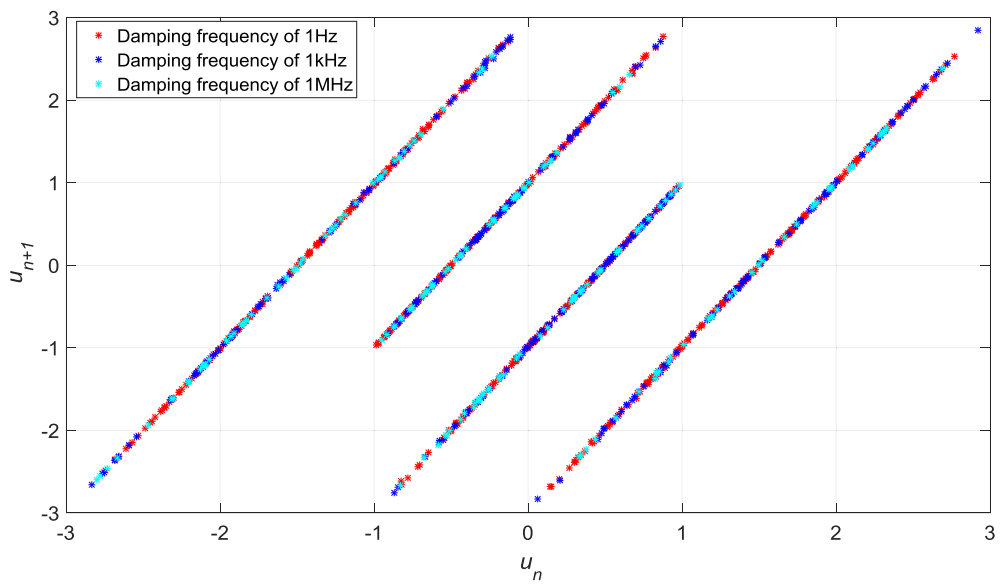

Fig. 10 A chaotic map of the proposed system with variable damping frequencies

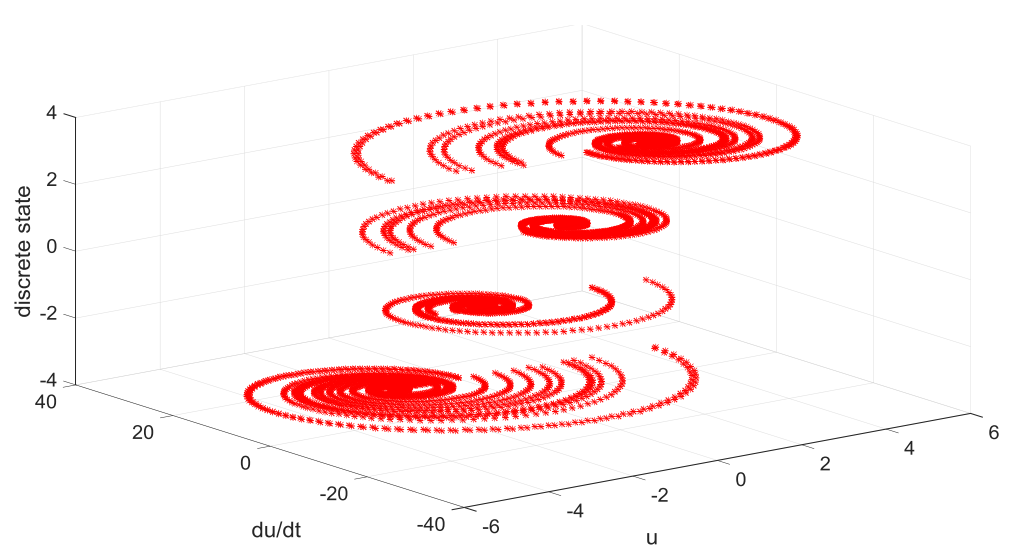

Fig. $113 D$ phase-space projection 


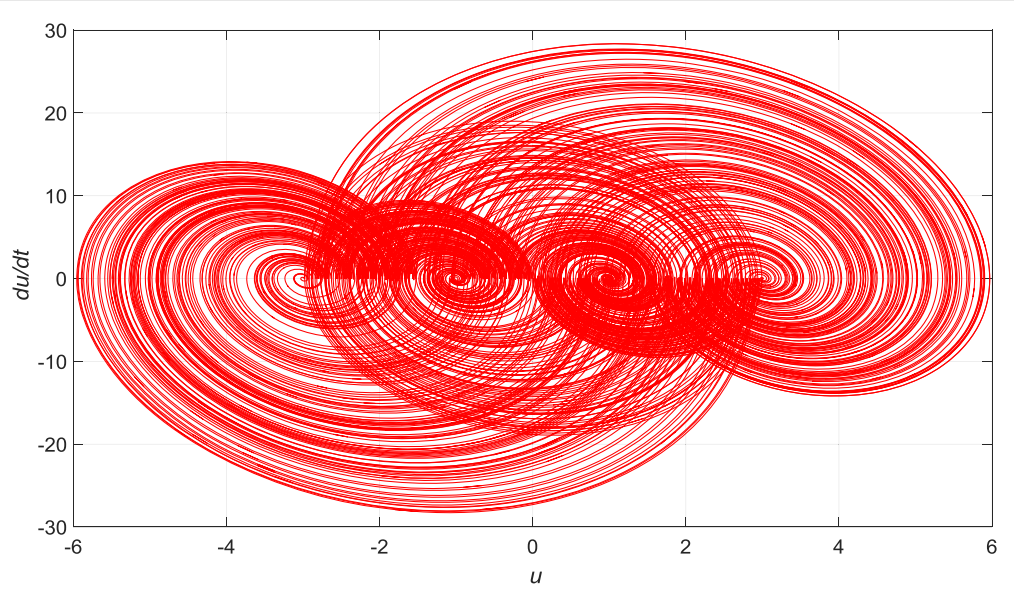

Fig. 12 2D phase-space projection

the generalized fixed basis function compared with a hybrid chaotic system based on forward time and DCSK. Therefore, it is proven that the GM-HCO for two levels outperforms that of a hybrid chaotic system based on forward time at approximately $1.2 \mathrm{~dB}$ of $\mathrm{Eb} / \mathrm{No}$ at $10^{-3}$ of BER, $3.2 \mathrm{~dB}$ of BFSK, and $5.7 \mathrm{~dB}$ of DCSK spreading by 10 chips, as well as providing performance approximating that of BPSK. Figure 14 shows the noise performance for four levels, using the proposed system based on GM-HCO compared with chaotic communication systems and a conventional system. It indicates that the proposed system provides improved performance in comparison to existing chaotic communication systems, including MCS-DCSK [46], $M$-ary FM-DCSK [47], $M$-ary OM-DCSK [21], QCSK [48] and MC-DCSK [49]. For consistency of comparison, all systems were chosen with four levels. Although increasing the number of levels leads to degradation of $\mathrm{GM}-\mathrm{HCO}$, the proposed system is more secure than periodic communication systems, such as $M$-ary PAM [50] due to its chaotic nature. In addition, because the proposed system is based on a coherent receiver, it is superior to chaotic systems based on a differentially coherent receiver in terms of bandwidth efficiency, power consumption, noise performance, data rate and simplicity. Finally, Fig. 15 shows that the noise performance is also not affected by variable damping frequencies $(1 \mathrm{~Hz}, 1 \mathrm{kHz}$, and $1 \mathrm{MHz})$.

\section{Discussion}

Since the GM-HCO is based on reverse time, a damping factor offers an opportunity to achieve variable throughput, higher operating frequency, low-power consumption, and low-cost to design compared with hybrid chaotic systems based on forward time. The cost and power

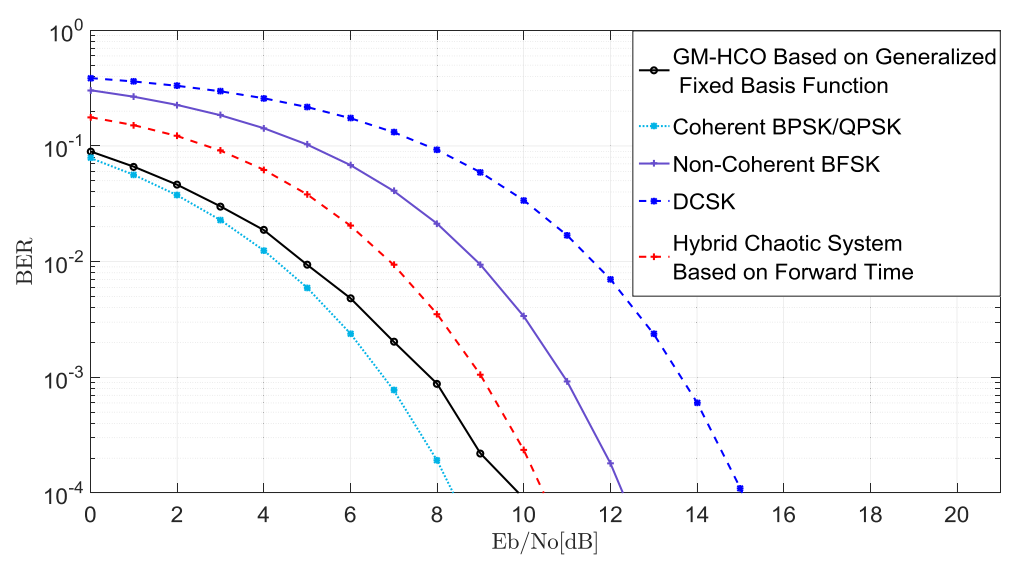

Fig. 13 BER performance of the proposed system based on GM-HCO for two levels over AWGN channel 


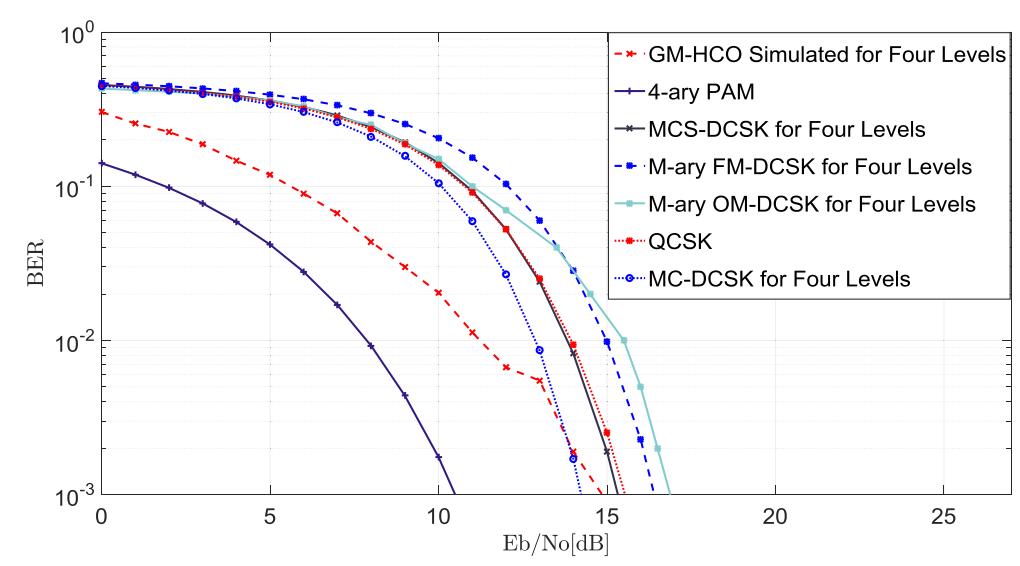

Fig. 14 BER performance of the proposed system based on GM-HCO over AWGN channel with four levels

consumption of systems based on GM-HCO are low because, as designed in [24], the hybrid chaotic system based on reverse time can be implemented using passive elements, such as resistors, capacitors, and inductors. By contrast, hybrid chaotic systems based on forward time need active elements, such as operational amplifiers [28]. Conventional chaotic systems also need active circuit elements [15]. Its dynamic map was created to examine the chaotic behavior of the differential equation of the oscillator in a multilevel approach. The novel generalized fixed basis function of the oscillator was then derived. Furthermore, the BER expression for two levels was derived to emphasize that the simulation matched theoretical outcomes. Simulation results indicated that the performance of the proposed GM-HCO based on a generalized fixed basis function provides improved performance compared with hybrid chaotic systems based on forward time, DCSK and some chaotic communication systems based on the $M$-ary concept and a differentially coherent receiver. It also provides comparable performance to conventional communication systems under AWGN channel conditions. Moreover, the results indicate that the proposed system provides lower power consumption, better bandwidth efficiency and data rate, and greater design simplicity. Although its noise performance will be degraded if the number of levels is increased, its transmitted signal is chaotic compared with periodic communication systems under AWGN.

\section{Conclusion}

This paper evaluated the noise performance, complexity, and power efficiency of an implementation of a chaotic communication system based on GM-HCO in a multilevel approach under AWGN channel conditions. The proposed hybrid chaotic oscillator offers a generalized framework to implement chaotic communication systems. The proposed system has flexible parameters such that it can be adopted for any communication scenario. Simulation and theoretical evaluations indicate that the systems based on the proposed oscillator outperform existing chaotic communication systems in terms of implementation complexity and

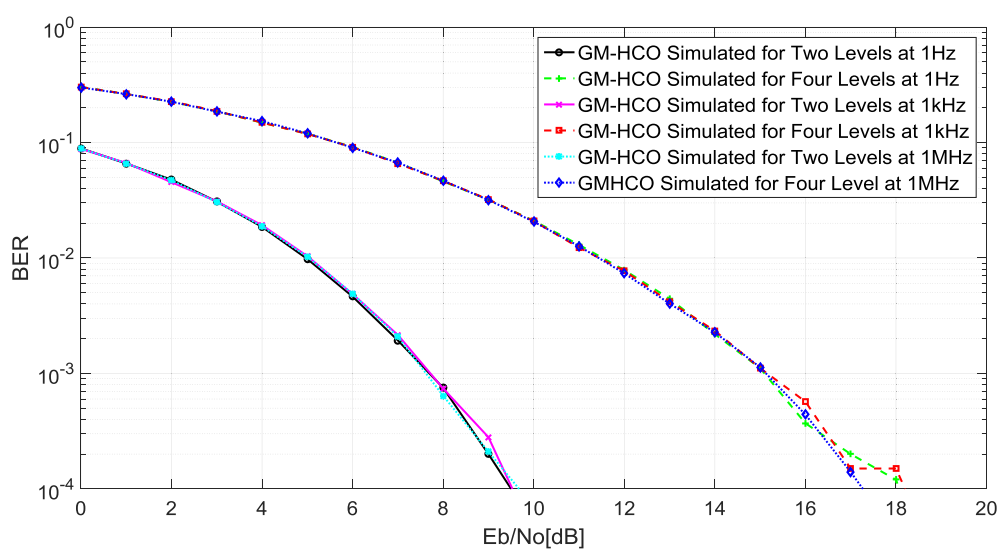

Fig. 15 BER performance of the proposed hybrid chaotic system over AWGN channel with variable frequencies 
bit-error-rate performance. Therefore, the proposed oscillator is a potential candidate for power efficient communications systems, such as wearable devices and IoT.

\begin{abstract}
Abbreviations
AWGN: Additive white gaussian noise; BER: Bit error rate; BFSK: Binary frequency shift keying; BPSK: Binary phase shift keying; CDMA: Code division multiple access; COOK: Chaotic on-off-keying; CSK: Chaos shift keying; DCSK: Differential chaos shift keying; FM-DCSK: Frequency modulation-differential chaos shift keying; GM-HCO: Generalized multilevel-hybrid chaotic oscillator; IOT: Internet of things; MC-DCSK: Multi-carrier differential chaos shift keying; MCS-DCSK: Multilevel code shifted-differential chaos shift keying; MIMO: Multiple input and multiple output; OM-DCSK: Orthogonal multilevel-differential chaos shift keying; PAM: Pulse amplitude modulation; PN: Pseudo-noise; QCSK: Quadrature chaos shift keying; SISO: Single input single output; UWB: Ultra-wide band
\end{abstract}

\section{Acknowledgements}

The first author would like to thank the Ministry of Higher Education and Scientific Research (MOHESR) in Iraq and Research Training Program (RTP) in Australia for funding his PhD scholarship.

\section{Authors' contributions}

$A D$ carried out the research with the help of DJ, PH, BS, and JB. DJ provided feedback, guidance, and research direction. PH helped with the mathematical formulation. BS helped with research direction and critical feedback. JB helped with the writing and reviewing. All authors read and approved the final manuscript.

\section{Authors' information}

Ammar Dukhan is a PhD candidate in chaos wireless communication engineering at QUT. He has a Bachelor's degree in electrical engineering and a Master's degree in secure communications engineering from Al-Mustansiriya University. He worked as a lecturer at the Electrical Engineering Department/ College of Engineering/ Al-Mustansiriya University/ Baghdad/ Iraq from 2005 until commencing his PhD studies at QUT in 2015.

Dr. Dhammika Jayalath, Senior Lecturer Science and Engineering Faculty, Queensland University of Technology (QUT). Dr Dhammika Jayalath received his PhD degree in Wireless Communication from Monash University, Australia. His research interest includes Channel Estimation, Communications Theory, Cognitive Radio/Cooperative Communications, Digital Communications, MIMO, Orthogonal Frequency Division Multiplexing (OFDM), Routing, Space-Time Signal Processing, synchronization, and Vehicle-To-Vehicle Communications.

A/Prof. Peter van Heijster is an applied analyst and his research focuses on nonlinear dynamics and in particular on understanding pattern formation. The aim of his research is to get a better understanding of the pattern formation processes in paradigmatic mathematical models and to apply the new insights to more biologically realistic models. He is particularly interested in the theoretical and mathematical underpinnings of the effects of heterogeneities on pattern formation processes.

Prof. Bouchra Senadji holds a Bachelor of Engineering in Electronics from ENSEEIHT, Toulouse, France, a Masters of Engineering from University Paul Sabatier, Toulouse, and a PhD in Signal Processing from Ecole Nationale Superieure des Telecommunications, Paris, France, which she received in 1992. She has worked as a Telecommunications Engineer at CNET, Paris, and is currently working at Queensland University of Technology, Brisbane, Australia, as an academic. Her areas of research are in Signal Processing applied to Telecommunications, including areas of MIMO, and Spectrum Sensing for Cognitive Radio.

Dr. Jasmine Banks is a Lecturer in the School of Electrical Engineering and Computer Science at Queensland University of Technology. She received her qualifications of B. Engineering (Electronics)/B. Information Technology from QUT in 1993, and PhD from QUT in 2000. Her current research interests include reconfigurable hardware, electronics, sensor fusion, computer vision, image processing, and signal processing.

\section{Funding}

Not applicable.
Availability of data and materials

Not applicable.

\section{Competing interests}

The authors declare that they have no competing interests.

Received: 2 September 2019 Accepted: 2 January 2020

Published online: 22 January 2020

\section{References}

1. (M. Eisencraft, R. Attux, R. Suyama, eds.), Chaotic signals in digital communications. (CRC Press, Boca Raton, 2013)

2. (H. Leung, ed.), Chaotic signal processing. (SIAM, Philadelphia, 2014)

3. H. Sompolinsky, A. Crisanti, H. J. Sommers, Chaos in random neural networks. Phys. Rev. Lett. 61(3), 259-262 (1988)

4. A. Babloyantz, A. Destexhe, Low-dimensional chaos in an instance of epilepsy. Proc. Nat. Acad. Sci. 83(10), 3513-3517 (1986)

5. M. V. Berry, Regularity and chaos in classical mechanics, illustrated by three deformations of a circular 'billiard'. Eur. J. Phys. 2(2), 91-102 (1981)

6. N. Tufillaro, A. Albano, Chaotic dynamics of a bouncing ball. Amer. J. Phys. 54(10), 939-944 (1986)

7. R. Walden, P. Kolodner, A. Passner, C. Surko, Traveling waves and chaos in convection in binary fluid mixtures. Phys. Rev. Lett. 55(5), 496-499 (1985)

8. E. Tziperman, L. Stone, M. A. Cane, H. Jarosh, El nino chaos: overlapping of resonances between the seasonal cycle and the pacific ocean-atmosphere oscillator. Science. 264(5155), 72-74 (1994)

9. E. N. Lorenz, Deterministic nonperiodic flow. J. Atmos. Sci. 20(2), 130-141 (1963)

10. J. M. González-Miranda, Synchronization and control of chaos: an introduction for scientists and engineers. (Imperial College Press, London, 2004)

11. E. Mosekilde, Y. Maistrenko, D. Postnov, Chaotic synchronization: applications to living systems. (World Scientific, Singapore, 2002)

12. S. C. Manrubia, A. S. Mikhailov, D. H. Zannette, Emergence of dynamical order: synchronization phenomena in complex systems. (World Scientific, Singapore, 2004)

13. I. Z. Kiss, J. L. Hudson, Chaotic cluster itinerancy and hierarchical cluster trees in electrochemical experiments. Chaos: Interdiscip. J. Nonlinear Sci. 13(3), 999-1009 (2003)

14. L. M. Pecora, T. L. Carroll, Synchronization in chaotic systems. Phys. Rev. Lett. 64(8), 821-825 (1990)

15. K. M. Cuomo, A. V. Oppenheim, Circuit implementation of synchronized chaos with applications to communications. Phys. Rev. Lett. 71(1), 65-68 (1993)

16. M. Kennedy, H. Dedieu, in Proc. Int. Workshop Nonlinear Dyn. Electron. Syst. (NDES" 93). Experimental demonstration of binary chaos-shift keying using self-synchronising chua's circuits (World Scientific, Dresden, 1993), pp. $67-72$

17. G. Kolumbán, B. Vizvári, W. Schwarz, A. Abel, in Proc. Int. Workshop Nonlinear Dyn. Electron. Syst. (NDES"96). Differential chaos shift keying: a robust coding for chaos communication (World Scientific, Seville, 1996), pp. $87-92$

18. G. Kolumbán, G. Kis, M. Kennedy, Z. Jákó, in Proc. Int. Symp. Nonlinear Theory Appl. FM-DCSK: a new and robust solution to chaos communications (IEICE (institute of electronics information and communication engineers), Hawaii, 1997), pp. 117-120

19. C. G. Rong, D. Xiaoning, From chaos to order: methodologies, perspectives and applications. (World Scientific, Singapore, 1998)

20. M. R. Casu, G. Durisi, Implementation aspects of a transmitted-reference UWB receiver. Wirel. Commun. Mobile Comput. 5(5), 537-549 (2005)

21. H. Yang, W. K. Tang, G. Chen, G. P. Jiang, System design and performance analysis of orthogonal multi-level differential chaos shift keying modulation scheme. IEEE Trans. Circuits Syst. I. 63(1), 146-156 (2016)

22. L. Wang, G. Cai, G. R. Chen, Design and performance analysis of a new multiresolution $M$-ary differential chaos shift keying communication system. IEEE Trans. Wirel. Commun. 14(9), 5197-5208 (2015)

23. H. Yang, W. K. Tang, G. Chen, G. P. Jiang, Multi-carrier chaos shift keying: system design and performance analysis. IEEE Trans. Circ. Syst. I. 64(8), 2182-2194 (2017)

24. N. J. Corron, S. T. Hayes, S. D. Pethel, J. N. Blakely, Chaos without nonlinear dynamics. Physical Rev. Lett. 97(2(024101)) (2006)

25. N. J. Corron, An exactly solvable chaotic differential equation. Dyn. Contin. Discrete Impuls. Syst.: Ser. A. 16, 777-788 (2009) 
26. O. E. Rössler, An equation for continuous chaos. Phys. Lett. A. 57(5), 397-398 (1976)

27. N. J. Corron, S. T. Hayes, S. D. Pethel, J. N. Blakely, Synthesizing folded band chaos. Phys. Rev. E. 75(4(045201)) (2007)

28. N. J. Corron, J. N. Blakely, M. T. Stahl, A matched filter for chaos. Chaos: Interdiscip. J. Nonlinear Sci. 20(2(023123)) (2010)

29. N. J. Corron, J. N. Blakely, Chaos in optimal communication waveforms. Proc. Roy. Soc. A: Math. Phys. Eng. Sci. 471(2180(20150222)) (2015)

30. N. J. Corron, J. N. Blakely, Controlling symbolic dynamics in an exact folded-band chaotic oscillator. Int. Fed. Autom. Control (IFAC) Proc. Vol. 45(12), 143-148 (2012)

31. N. J. Corron, J. N. Blakely, Exact folded-band chaotic oscillator. Chaos: Interdiscip. J. Nonlinear Sci. 22(2(023113)) (2012)

32. N. J. Corron, J. N. Blakely, in Proc. Nonlinear Theory Appl. (NOLTA). Exactly solvable chaos as communication waveforms (IEICE (Institute of Electronics Information and Communication Engineers), Santa Fe, 2013), pp. 217-220

33. D. W. Hahs, N. J. Corron, J. N. Blakely, Synthesizing antipodal chaotic waveforms. J. Frankl. Inst. 351(5), 2562-2573 (2014)

34. N. J. Corron, J. N. Blakely, V. In, P. Longhini, A. Palacios, in Amer. Inst. Phys. (AIP) Conf. Proc., vol. 1339. A matched filter for communicating with chaos (AIP, Alberta, 2011), pp. 25-35

35. M. S. Milosavljevic, J. N. Blakely, N. J. Corron, in Proc. 14th Int. Conf. Appl. Nonlinear Dyn. (ICAND). A solvable chaotic oscillator with multiple set points (Springer, Denver, 2016), pp. 33-39

36. N. J. Corron, R. M. Cooper, J. N. Blakely, Analytically solvable chaotic oscillator based on a first-order filter. Chaos: Interdiscip. J. Nonlinear Sci. 26(2(023104)) (2016)

37. H.-P. Ren, C. Bai, Q. Kong, M. S. Baptista, C. Grebogi, A chaotic spread spectrum system for underwater acoustic communication. Physica A: Stat. Mech. Appl. 478, 77-92 (2017)

38. A. Beal, J. Blakely, N. Corron, R. Dean, in Proc. SPIE Radar Sensor Technol., vol. 9829. High frequency oscillators for chaotic radar (SPIE Conf., Baltimore, 2016)

39. N. J. Corron, M. T. Stahl, R. Chase Harrison, J. N. Blakely, Acoustic detection and ranging using solvable chaos. Chaos: Interdiscip. J. Nonlinear Sci. 23(2(023119)) (2013)

40. J. Bailey, A. Beal, R. Dean, M. Hamilton, J. Tugnait, High-frequency reverse-time chaos generation using digital chaotic maps. Electron. Lett. 50(23), 1683-1685 (2014)

41. N. J. Corron, S. T. Hayes, S. D. Pethel, J. N. Blakely, in 2007 IEEE Int. Symp. Circuits Syst. Reverse-time chaos from a randomly driven filter (New Orleans, IEEE, 2007), pp. 205-208

42. J. P. Bailey, A. N. Beal, R. N. Dean, M. C. Hamilton, A digital matched filter for reverse time chaos. Chaos: Interdiscip. J. Nonlinear Sci. 26(7(073108)) (2016)

43. S. H. Strogatz, Nonlinear dynamics and chaos: with applications to physics, biology, chemistry, and engineering. (CRC Press, Boca Raton, 2014)

44. J. Bailey III, Digital reverse time chaos and matched filter decoding. Ph.D. dissertation, Dept. Elect. Comput. Eng., Auburn Univ., Auburn, AL, USA, (2015). http://etd.auburn.edu/handle/10415/4544. Accessed Jan 2019

45. W. Szemplinska-Stupnicka, Chaos bifurcations and fractals around us: a brief introduction. (World Scientific, Singapore, 2003)

46. T. Huang, L. Wang, W. Xu, F. C. Lau, Multilevel code-shifted differential-chaos-shift-keying system. IET commun. 10(10), 1189-1195 (2016)

47. G. Kis, Performance analysis of chaotic communications systems. Ph.D. dissertation, BME-MIT, Budapest, Hungary (2003). Available: https:// repozitorium.omikk.bme.hu/bitstream/handle/10890/296/ertekezes.pdf Accessed Jan 2019

48. Z. Galias, G. M. Maggio, Quadrature chaos-shift keying: theory and performance analysis. IEEE Trans. Circ. Syst. I: Fundam. Theory Appl. 48(12), 1510-1519 (2001)

49. G. Kaddoum, F.-D. Richardson, F. Gagnon, Design and analysis of a multi-carrier differential chaos shift keying communication system. IEEE Trans. Commun. 61(8), 3281-3291 (2013)

50. M. Safak, Digital communications. (John Wiley \& Sons, Chichester, 2017)

\section{Publisher's Note}

Springer Nature remains neutral with regard to jurisdictional claims in published maps and institutional affiliations.

\section{Submit your manuscript to a SpringerOpen ${ }^{\circ}$ journal and benefit from:}

- Convenient online submission

Rigorous peer review

- Open access: articles freely available online

- High visibility within the field

- Retaining the copyright to your article

Submit your next manuscript at $>$ springeropen.com 